\title{
ENTERPRISE EVOLUTION STRATEGY ANALYSIS BY UNEARTHING SUPERIORITY IN ANP SUPERMATRIX
}

\author{
Xing Yin \\ School of Automation \\ Huazhong University of Science and Technology \\ Wuhan, Hubei, P.R.China \\ E-mail: yxx0906@163.com \\ Xiaomeng Yin* \\ School of Automation \\ Huazhong University of Science and Technology \\ Wuhan, Hubei, P.R.China \\ E-mail: yin90m@qq.com
}

\begin{abstract}
The process of reaching the limit of supermatrix can be considered as the accumulation of index effect, which is impliedly infected to the evolution of the enterprise architecture model. And then, the gradually increasing sequence step of supermatrix's power can be treated as an indicator of interaction of the indexes in the evolution strategy. With an ANP supermatrix, the influence of complex factors in the evolution of enterprise strategy is researched based on the architecture model, and an ANP analytical method to parse the effective factors in the evolution of enterprise strategy is put forward. We apply the ANP analytical method of the influential factors in enterprise evolution strategy to the cloud migration evolution strategy of a business park system. By finding the key influential factors acting to improve system development plan, the method proves to be effective that it improves the cloud migration strategy of enterprise information system, as well as adjusts the cloud resource consolidation towards a better and more balanced performance.
\end{abstract}

Keywords: ANP, supermatrix sequence, cumulative influence change, next key factor, enterprise evolution strategy

\section{Introduction}

Enterprise architecture is as strategy (Ross, Weill, \& Robertson, 2006). Enterprise architecture describes and defines an enterprise's vision, target, organization, ability and roles, acting as a best guide to turn enterprise evolution strategy into practice. With the ANP model, we can build the business model, data model, application model and technology model properly, and evaluate the static overall enterprise system.

Since the theory and method of ANP (Saaty, 1996) was first systematically put forward, ANP has been widely applied and researched in the business areas: economy forecasts (Blair, Nachtmann \& Saaty, 2002), energy strategy plan (Ulutas \& B.H, 2005), industrial management (Wolfslehner, Vacik \& Lexer, 2005), etc. The successful applications of these aspects fully illustrate the effectiveness of applying ANP to complicated multi-criteria decision-making in enterprise strategy management. However, these

\footnotetext{
* Corresponding author

Project supported by the National Nature Science Foundation of China (No. 61273207 )
} 
decisions can only be effective at a certain time point. The research of a scientific way to support the enterprise evolution strategy in the long run is thus heating up. Enterprise Architecture Analysis using AHP and Fuzzy AHP (Davoudi \& Sheykhyand, 2011) provides an approach with AHP towards enterprise architecture analysis. Research on the evolution of enterprise clusters based on theory of MAS (Xu, Liu \& Shao, 2011) simulates the evolution of enterprise clusters by a multi-agent model using the theory of MAS and the AE model. Other papers, such as Research on Mechanism of Enterprise Evolution Based on Biological Evolution (Ye \& Zhang, 2012) and Research on Evolution and Evaluation of Construction Enterprise based on the Dissipative Structure Theory (Yan, 2012), try to analyze the enterprise evolution in different fields.

This paper combines the enterprise architecture model with Analytic Network Process (ANP) to build enterprise strategy evaluation model, where Super Decisions tool is employed to facilitate ANP supermatrix calculation. To explore the interaction of complex factors in the enterprise evolution based on the architecture model, we innovatively put forth an ANP analytical method to resolve the influence on enterprise development plan-ANP differential matrix sequence analysis method. It takes the interactions of systems and the gradual changes of influence into account. And eventually we can find out the key direct and indirect factors that impact on a certain factor at different stages of enterprise evolution, solving the challenges and bottlenecks in the development of enterprises.

\section{Differential matrix sequence analysis method based on ANP}

The ANP initial weighted supermatrix $W$ reflects the direct influence between elements, and the limit supermatrix shows the final consequences of interactions between elements. These two matrixes demonstrate the importance of each element in a static state, including the initial state and the final state of elements' interaction. Hence the method based on ANP is proposed to capture the change in the importance of each index in the process from initial supermatrix to limit supermatrix. Using this method, the vector sequence including important elements in stages can be obtained, and we label it as $K(i)=\{$ key factor in current stage $e_{j}$, key directly influence factor $e_{k}$, key indirectly influence factor $e_{l}$, key factor in next stage $\left.e_{n}\right\}$. This sequence can be used for revising the strategic evolution plan of enterprise. Specific steps are as follows.

\subsection{Obtain key element in initial stage}

When ANP is used to address decision problems, it has the network structure with both internal dependence and external dependence differing from the hierarchical structure in AHP. The element preference rank, affected by the entire network, can be obtained based on the importance of the relative influence of other elements when a certain element acts as a criterion. According to the limit state of the influence between the elements in the ANP network, we take the limit supermatrix to calculate the absolute order of the elements' importance dominance of the system.

The influence structure of ANP network contains the elements' external and internal periodic interactions, which exists in all the supermatrix of different power. Accordingly, the influence structure between the elements is divided into the following two basic forms in Figure 1. 

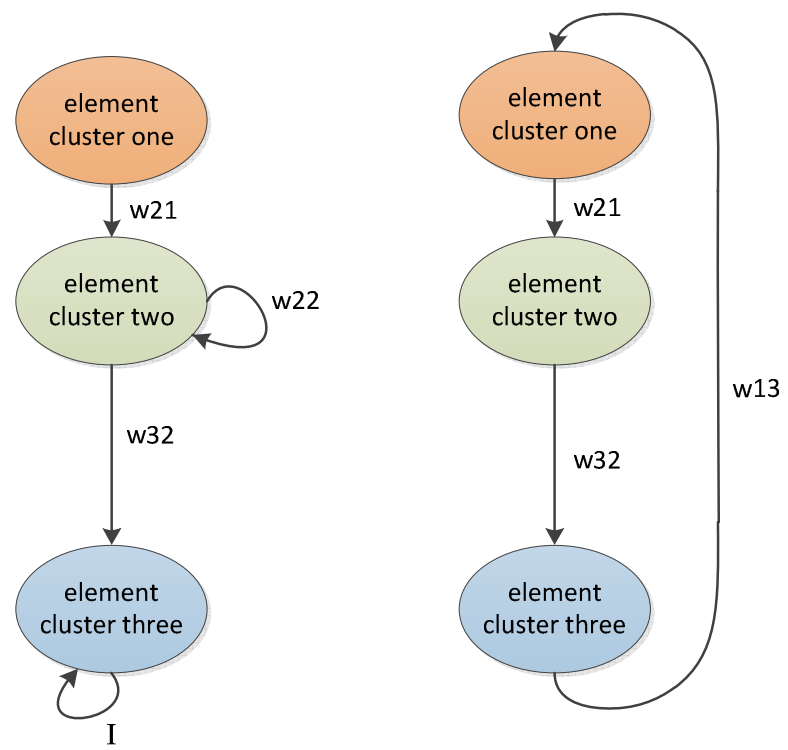

Figure 1. Element's external and internal periodic interaction.

ANP differential matrix sequence analysis method will get the same results from these two types of basic structures; this paper will analyze and illustrate the first structure by this method. The ANP limit Supermatrix $W^{\infty}$ reflects the overall result of total influence which contains the absolute rank of element importance, and $W^{\infty}$ has the same column vectors. For a column vector $\mathrm{V}$ in $W^{\infty}$, by observing the maximum weight value, the key factor of the evolution strategic is found, and it will be treated as element $e_{i 1}$ to be improved at the initial stage.

\subsection{Obtain the key direct and indirect factor}

The limit supermatrix shows the final result of influence transmitted by all the elements' interactions in the ANP model. The value $w_{i j}$ in the weighted supermatrix denotes the direct influence between elements $i$ and $j$, however, the indirect influence cannot be ignored. When the influence is transmitted from element $\mathrm{i}$ to element $\mathrm{j}$ through all the possible single middle element $\mathrm{k}$, the sum of the indirect influence is calculated as $W^{2}$ with its value $w_{i j}^{2}=\sum w_{i k} w_{k j}$. Similarly, $W^{k}$ defines the sum of the indirect influence values when the influence is transmitted from element $i$ to element $j$ through all of the possible $k-1$ middle elements, so $W^{k}$ is the direct interaction matrix in the $\mathrm{k}$ stage.

Analyzing the weighted supermatrix $W$ and the kth power of supermatrix $W$ of the first ANP network structure, they have the following forms:

$$
W=\left(\begin{array}{ccc}
0 & 0 & 0 \\
w_{21} & w_{22} & 0 \\
0 & w_{32} & I
\end{array}\right) \quad W^{k}=\left(\begin{array}{ccc}
0 & 0 & 0 \\
w_{22}{ }^{k-1} w_{21} & w_{22}{ }^{k} & 0 \\
w_{32} \sum_{i=0}^{n-2} w_{22}{ }^{i} w_{21} & w_{32} \sum_{i=0}^{n-1} w_{22}{ }^{i} & I
\end{array}\right)
$$


The direct influence caused by the first stage of elements' interactions is implied in the initial supermatrix $\mathrm{W}$, and the kth power of supermatrix implies elements' direct influence in stage $\mathrm{k}$. For the element cluster three, namely $\mathrm{C} 3$, the effects in different stages are shown as Figure 2 as below:

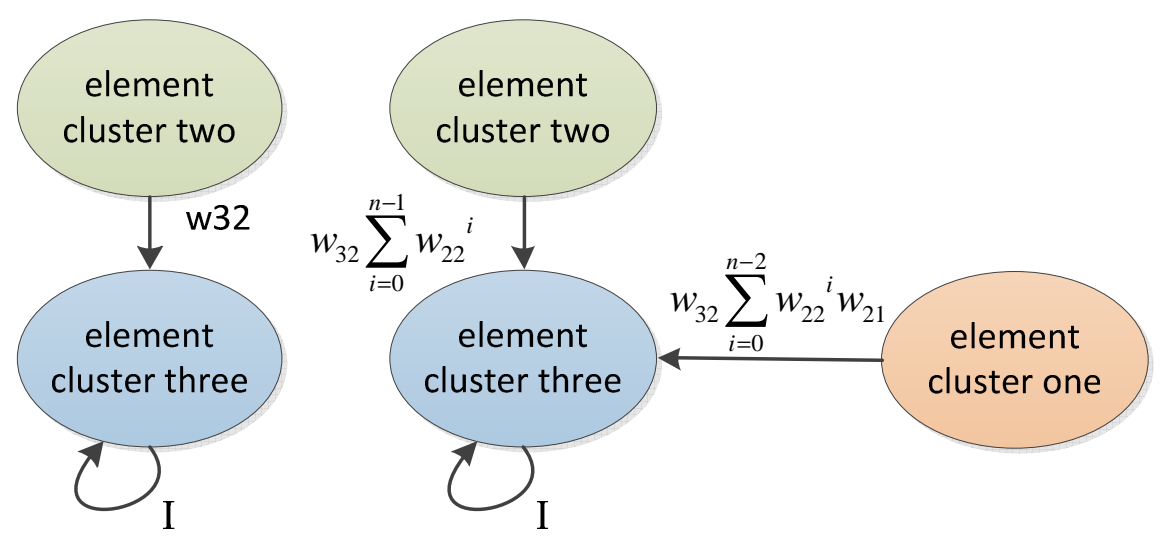

Figure 2. Changed influence in different stage to C3 (cluster three).

The sequence of power matrixes shows the iterative process of interaction. For the elements in the same column of matrix $W^{*}$, each value represents the influence from the corresponding element in row to the element in column in the $\mathrm{k}$ stage, and the sum of the values in each column is one. For the column $\mathrm{j}$, $w_{k j}=\max w_{i j}$ represents the element which has the heaviest influence on the element $e_{j}$, named key directly influence factor $e_{k} ; w_{l k}=\max w_{i k}$ represents the element which has the heaviest influence on $e_{k} ;$ meanwhile it has the heaviest indirect influence on $e_{j}$, so we call it key indirectly influence factor.

The important elements unearthed from the matrixes, including the key factor $e_{j}$ in current stage, key directly influence factor $e_{k}$ and key indirectly influence factor $e_{l}$, indicate the direction of current state. These elements can be used to revise the enterprise plan: key factor in current stage points out the critical point of current construction, and all the strategic resources should focus on this point; key directly influence factor affects the quality of key construction point in the largest extent, and key indirectly influence factor similarly has the heaviest influence indirectly on the key point, so both effects on current construction of the entire enterprise cannot be ignored.

\subsection{Approach improving factor in next stage by ANP differential matrixes sequence}

We use the sequence $W, W^{2}, W^{3}, \cdots, W^{k}, \cdots, W^{\infty}$ to study the gradual interaction of elements' influence at different stages. In the process of supermatrix's power gradual growth, the structure and weights of the effects in the ANP model changes in different phases. And the process to get the limit supermatrix, $W^{k}$ includes the information of the superiority degrees in the elements' interaction. By the minus of one supermatrix in the sequence to the adjacent supermatrix, which can be expressed as $\Delta W_{k-1}=W^{k}-W^{k-1}$, we analyze the changes of elements' influence in supermatrixes which are in the sequence order. And we can get the influence changes of all the elements to a specific element in a certain stage interval, by making the other elements as the criteria. The differential matrix sequence is as follows: 


$$
\begin{aligned}
& \Delta W_{1}=W^{2}-W \\
& \Delta W_{2}=W^{3}-W^{2} \\
& \Delta W_{3}=W^{4}-W^{3} \\
& \cdots \\
& \Delta W_{k-1}=W^{k}-W^{k-1}
\end{aligned}
$$

Taking the first ANP network model for analysis, we compare the supermatrix $W^{k}$ and $W^{k-1}$ to get the difference value between them:

$$
W^{k}-W^{k-1}=\left(\begin{array}{ccc}
0 & 0 & 0 \\
\left(w_{22}^{k}-w_{22}^{k-1}\right) w_{21} & w_{22}^{k}-w_{22}^{k-1} & 0 \\
w_{32} w_{22}^{k-2} w_{31} & w_{32} w_{22}^{k-1} & 0
\end{array}\right)
$$

For the element cluster of $\mathrm{C} 3, W^{k}-W^{k-1}$ compares the influence in the $\mathrm{K}$ stage implied in the supermatrix with the influence in the K-1 stage implied in the supermatrix $W^{k-1}$ to find the influence changes in the network structure, which is showed in Figure 3:

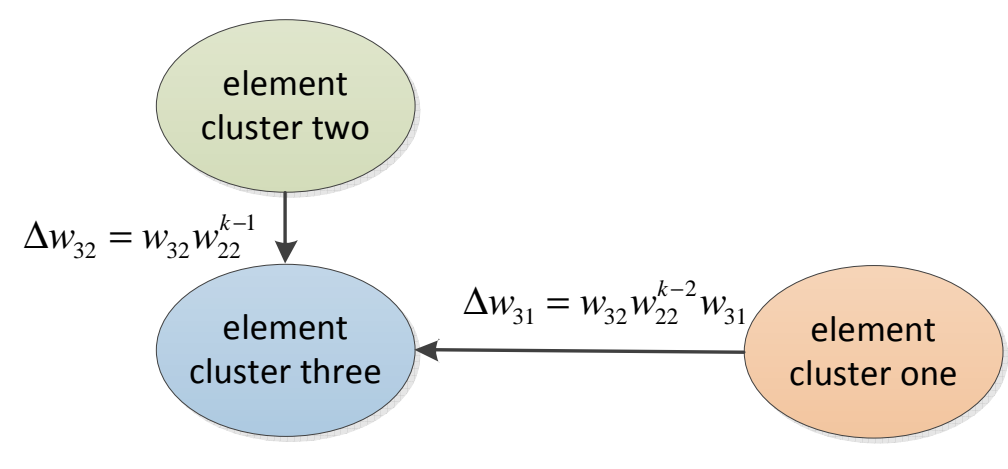

Figure 3. Changed influence from current stage to next stage.

In order to make the analysis easier, taking element $\mathrm{A}$ as a criterion, there are influence $p_{A}\left(C 1_{i}\right)$ and $p_{A}\left(C 1_{j}\right)$ between elements $C 1_{i}, C 1_{j}$ and A. So the dominance between $p_{A}\left(C 1_{i}\right)$ and $p_{A}\left(C 1_{j}\right)$ can be expressed by $p_{A}\left(C 1_{i}\right)<p_{A}\left(C 1_{j}\right), \quad p_{A}\left(C 1_{i}\right)>p_{A}\left(C 1_{j}\right)$ and $p_{A}\left(C 1_{i}\right)=p_{A}\left(C 1_{j}\right)$ when $p \in[0,1]$. In the gradual process from the matrix $W^{k-1}$ to the matrix $W^{k}$, the change of influence from element $C 1_{j}$ to element $\mathrm{A}$ can be written as $\Delta p_{A}\left(C 1_{j}\right)$. The kth stage of influence from element $C 1_{j}$ to element $\mathrm{A}$ is expressed as $S_{A}^{k}\left(C 1_{j}\right) \cdot \Delta p_{A}^{k-1}\left(C 1_{j}\right)=p_{A}^{k}\left(C 1_{j}\right)-p_{A}^{k-1}\left(C 1_{j}\right)$ means the influence changed from stage $\mathrm{k}$ to stage k-1. When $S_{A}\left(C 1_{j}\right) \in\left[S_{A}^{k-1}\left(C 1_{j}\right), S_{A}^{k}\left(C 1_{j}\right)\right]$, we put all the other elements' influence to a certain element 
together, which reflects the changes of influence dominant in the k-1 phase. Take element cluster 3 as the certain element.

$$
\begin{aligned}
& \text { When } S_{C 3} \in\left[S_{C 3}^{k-1}, S_{C 3}^{k}\right],\left\{\begin{array}{c}
\Delta p_{C 1}^{k-1}(C 3)=p_{C 1}^{k}(C 3)-p_{c 1}^{k-1}(C 3)=w_{32} w_{22}^{k-2} w_{31} \\
\Delta p_{C 2}^{k-1}(C 3)=p_{C 1}^{k}(C 3)-p_{c 1}^{k-1}(C 3)=w_{32} w_{22}^{k-1}
\end{array}\right. \\
& \text { Then } e_{n}=\Delta p_{C 3}^{k-1}\left(C_{j}\right)=\max \left\{\begin{array}{c}
\left.\Delta p_{C 3}^{k-1}\left(C_{1}\right), \Delta p_{C 3}^{k-1}\left(C_{2}\right), \cdots, \Delta p_{C 3}^{k-1}\left(C_{j}\right), \cdots\right\}
\end{array}\right.
\end{aligned}
$$

Therefore, when $S_{c 3} \in\left[S_{c 3}^{t-1}, S_{c 3}^{t}\right]$, namely in the transition to the next stage $\mathrm{k}$, the key influence to element C3 is $e_{n}=\Delta p_{c 3}^{k-1}\left(C_{j}\right)$, which has an maximum weight. It is the jth vector in the matrix $\Delta W_{k-1}=W^{k}-W^{k-1}$, and it is the element to improve in next stage. The element found in this method has a crucial influence on the current stage's key element in the gradual changing process. Namely it will play a key role in the next step in the construction of the enterprise, so it needs to be taken into account in evolution plan as a key in next step of construction, and it can revise the evolution strategy in next step.

\subsection{Rank vector sequence of evolution scheme}

Back to step 2, more vector sequences K(i) can be achieved, until the changes of influence are less than the threshold $\delta$. The reason why $\delta$ exists is obvious: $w_{i j}=p_{j}(i) \in[0,1]$ in the initial supermatrix $\mathrm{W}$, so we can get the result of $\Delta p_{c 3}^{k-1}(C 1) \rightarrow 0$ and $\Delta p_{c 3}^{k-1}(C 2) \rightarrow 0$ when $s_{c 3} \in\left[s_{c 3}^{k-1}, s_{c 3}^{k}\right]$ and $\mathrm{k} \rightarrow \infty$. Using $\Delta P \in[\delta, 1]$ as the obvious scope of influence change judged by decision maker, the schemes for evolution can be worked out till reaching stage $\mathrm{k}$. When $\Delta p \in[\delta, 1]$ and $s_{i} \in\left[s^{\prime}, s^{2}\right],\left[s^{2}, s^{3}\right], \cdots,\left[s^{t-1}, s^{\prime}\right]$, there are vector sequences in different phases: $\mathrm{K}(1), \mathrm{K}(2), \ldots, \mathrm{K}(\mathrm{k}-1)$. This sequence acts as the basis to develop the evolution strategy which promotes the system performance.

\section{A cloud migration evolution strategy for an enterprise}

In this paper, we apply our method to a cloud migration evolution strategy of a business park information system. Using this example, steps will be described in detail about how to combine the ANP with enterprise evolution to get a strategic roadmap for enterprise development.

Before obtaining the metrics of evaluating the adequacy of system migration, we analyzed the business process for the park system and established the architecture model. Then an ANP evaluation model for the construction of private park cloud was built in SuperDecisions. In the model, we only focused on the interaction between evaluation indexes, but ignored the alternatives' influence on the indexes.

The evaluation model extracted a total of 17 indexes from four aspects, so that we can evaluate the adequacy of system migration comprehensively. We first acquired the subjective preference information from decision makers, and then constructed the corresponding judgment matrix. The weighted supermatrix and limited supermatrix were generated automatically from SuperDecisions. Thus we can use them to produce an ANP differential sequence to make an enterprise development process plan. The ANP model is shown in Figure 4 as below: 


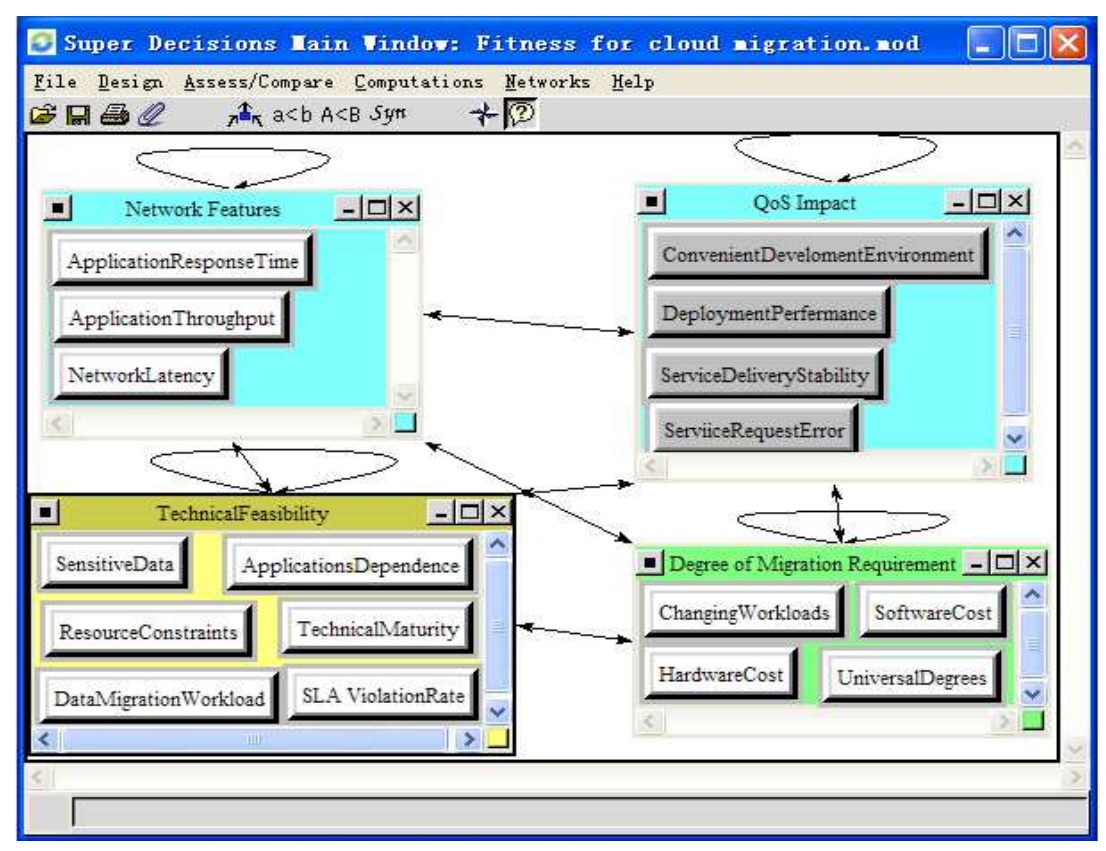

Figure 4. ANP model of system migration adequacy.

By analyzing the limit supermatrix and cloud migration steps of the enterprise system, the element with maximum weight, namely data migration workload is found. It means that enterprise strategy should focus on that factor at the initial stage. Following the method mentioned above, next step is to find the key direct and indirect influence elements for data migration workload in the weighted supermatrix. The weighted supermatrix $W$ exported from SuperDecisions is shown in Table 1.

Table 1. The weighted supermatrix $W$ of cloud migration

\begin{tabular}{|c|l|l|l|l|l|l|}
\hline & \multicolumn{1}{|c|}{ SVR } & \multicolumn{1}{c|}{ AD } & \multicolumn{1}{c|}{ TM } & \multicolumn{1}{c|}{ SD } & \multicolumn{1}{c|}{ DMW } & \multicolumn{1}{c|}{ RC } \\
\hline ART & 0.062271 & 0.004483 & 0 & 0.012919 & 0.02824 & 0.041572 \\
\hline AT & 0.055415 & 0.011749 & 0.043741 & 0.023475 & 0.06558 & 0.038002 \\
\hline NL & 0.094484 & 0.002566 & 0 & 0.042657 & 0.092454 & 0.032086 \\
\hline DEC & 0 & 0.022254 & 0.085903 & 0 & 0.057269 & 0.01993 \\
\hline SDS & 0.022491 & 0.077854 & 0 & 0.075796 & 0.025463 & 0.020699 \\
\hline SRE & 0.034026 & 0.233584 & 0 & 0.217257 & 0.010324 & 0.048247 \\
\hline DP & 0 & 0.011127 & 0.056719 & 0 & 0.028635 & 0.029898 \\
\hline SVR & 0.028129 & 0 & 0 & 0 & 0.047225 & 0.021799 \\
\hline AD & 0.10278 & 0.108544 & 0.417136 & 0.074003 & 0.299688 & 0.06078 \\
\hline TM & 0.047467 & 0.005023 & 0.162636 & 0.033736 & 0.040829 & 0.013415 \\
\hline SD & 0.068705 & 0 & 0.007042 & 0.021325 & 0.007554 & 0.030384 \\
\hline DMW & 0.19647 & 0.0401 & 0.119311 & 0.047908 & 0.115025 & 0.26206 \\
\hline RC & 0.215677 & 0.003503 & 0.034483 & 0 & 0.129135 & 0.159517 \\
\hline WC & 0.049238 & 0.159738 & 0.032948 & 0.165928 & 0.039434 & 0.060667 \\
\hline HC & 0 & 0 & 0.012337 & 0 & 0 & 0 \\
\hline SC & 0 & 0 & 0.008688 & 0 & 0 & 0 \\
\hline UD & 0.022845 & 0.319477 & 0.019056 & 0.284997 & 0.013145 & 0.160946 \\
\hline
\end{tabular}


Note1. $\mathrm{ART}=$ application respond time; $\mathrm{AT}=$ application throughput $\mathrm{NL}=$ network latency; $\mathrm{DEC}=$ development environment convenient; SDS = service delivery stability; SRE = service request error; DP $=$ deployment performance; SVR = SLA violation rate; $\mathrm{AD}=$ application dependence; $\mathrm{TM}=$ technical maturity; $\mathrm{SD}=; \mathrm{DMW}=$ data migration workload; $\mathrm{RC}=$ resource constraints; $\mathrm{WC}=$ workloads change; $\mathrm{HC}=$ hardware cost $; \mathrm{SC}=$ software cost $\mathrm{UD}=$ universal degree;

Note2. The row just contains the factors involved in the discussion in the following content.

We get the maximum value in the column where the data migration workload is the criterion. It is the applications dependence. It indicates that applications dependence has the maximum direct influence on the data migration workload initially, so we call it the key direct influence factor at the present stage. Then, in the column which takes the applications dependence as criterion, the factor which has the maximum value is universal degree. So, universal degree has the heaviest influence on the factor of applications dependence. The influence transferred from applications dependence to data migration workload represents the indirect influence of universal degree to data migration workload. So the universal degree is called the key indirect influence factor.

Now the key factor in stage 2 needs to be found out. For this purpose, we should analyze the difference between $W$ and $W^{2}$. The matrix $\Delta W_{1}=W^{2}-W$ is shown in the Table 2 below. The universal degree increases most in the column where the data migration workload is chosen as criterion.

Table 2. The supermatrix $\Delta W_{1}$ of cloud migration.

\begin{tabular}{|c|l|l|l|l|l|l|}
\hline & \multicolumn{1}{|c|}{ SVR } & \multicolumn{1}{c|}{ AD } & \multicolumn{1}{c|}{ TM } & \multicolumn{1}{c|}{ SD } & \multicolumn{1}{c|}{ DMW } & \multicolumn{1}{c|}{ RC } \\
\hline ART & 0.004159 & 0.019765 & 0.009105 & -0.01833 & 0.01003 & 0.001419 \\
\hline AT & -0.0053 & 0.032913 & -0.01859 & 0.002253 & 0.0308 & 0.017924 \\
\hline NL & 0.006028 & 0.029675 & -0.05154 & 0.018634 & 0.021965 & -0.02371 \\
\hline DEC & -0.06185 & 0.044823 & -0.05005 & 0.004642 & 0.015551 & -0.02649 \\
\hline SDS & 0.012798 & -0.05192 & -0.00711 & 0.016093 & 0.019283 & 0.013174 \\
\hline SRE & 0.037349 & -0.19429 & 0.039848 & 0.04558 & 0.043266 & 0.043702 \\
\hline DP & -0.00365 & 0.029056 & -0.01098 & -0.01288 & -0.00847 & -0.07549 \\
\hline SVR & 0 & 0.03875 & -0.03489 & -0.01078 & 0.008217 & 0.005484 \\
\hline AD & -0.36672 & 0.14605 & -0.23753 & 0.100335 & 0.163759 & 0.057518 \\
\hline TM & -0.10348 & -0.00205 & 0.0012 & 0.031346 & 0.040394 & 0.034413 \\
\hline SD & 0.00073 & -0.00616 & 0.03572 & -0.006 & 0.00538 & -0.00981 \\
\hline DMW & 0.241947 & 0.116129 & 0.171976 & -0.11867 & -0.19262 & 0.003611 \\
\hline RC & 0.018335 & 0.139262 & 0.005513 & -0.05818 & -0.11116 & 0.062291 \\
\hline WC & 0.030714 & -0.11942 & 0.027626 & 0.027011 & -0.00172 & 0.034773 \\
\hline HC & -0.00912 & 0 & 0.001345 & 0.001405 & 0.001184 & 0.002373 \\
\hline SC & -0.00622 & 0 & 0.000947 & 0.000989 & 0.000834 & 0.003949 \\
\hline UD & 0.204284 & -0.22259 & 0.117412 & -0.02345 & -0.04669 & -0.14512 \\
\hline
\end{tabular}

Note1. The note of this table is the same as Table 1.

We can take previous steps to gain the crucial indicator vector $K(1)=\{$ data migration workload, applications dependence, universal degree, universal degree \}. This indicator vector will be an initial stage guidance to plan the system evolution: Treating the data migration workload as the current key element, the enterprise can make reasonable plans to migrate the system data into the cloud platform. 
Then it needs to distinct between public and private data to avoid repeated work of data migration; Applications dependence acts as the most important direct influence factor to influence the current key index - data migration workload, and it plays a crucial role in the whole cloud computing system construction. It is necessary to consider the collaborative characteristics of the migration of highly dependent application system, avoiding to migrate the applications with strong connections to each other, in order that coupling performance of the whole system is maintained at a high level; the universal degree is a key indirect factors, acting as an important auxiliary role in construction, which needs to be planed reasonably; finally, the universal degree is also viewed as a key factor to be focused on in the next stage. Considering the development in the long-term, the current plan also needs to be carried out around this element to ensure a smooth transition to the next stage.

$\mathrm{K}(2)=$ universal degree, service request error, data migration workload, universal degree $\}$

$\mathrm{K}(3)=$ \{universal degree, resource constraints, data migration workload, universal degree $\}$

$\mathrm{K}(4)=\{$ universal degree, data migration workload, applications dependence, applications dependence $\}$

$\ldots$

The sequence of important element vector of the above offers some tips for enterprise to make development plan in different stages. One of them is that some elements, such as applications dependence, appear repeatedly, illustrating that some key factors are considered to be the key point in a certain evolution phrase of enterprise system migration; The other is that some elements appear as key elements in successive phases, such as universal degree, indicating that this kind of elements is critical to the performance of the cloud migration in a long process and needs to pay constant attention to them.

By gradually extracting the sequence of important element vectors $\mathrm{K}(\mathrm{x})(\mathrm{x}=1,2, \cdots, \infty)$ to conduct the enterprise development at different stages following the steps above, we can identify the key goals to establish enterprises evolution strategy .It wise to combine the important elements in following phrases with park business project, which can supplement and perfect the plan of cloud migration of the entire park, and finally formulate a phased enterprise evolution. The cloud migration evolution of the enterprise is showed in Figure 5.

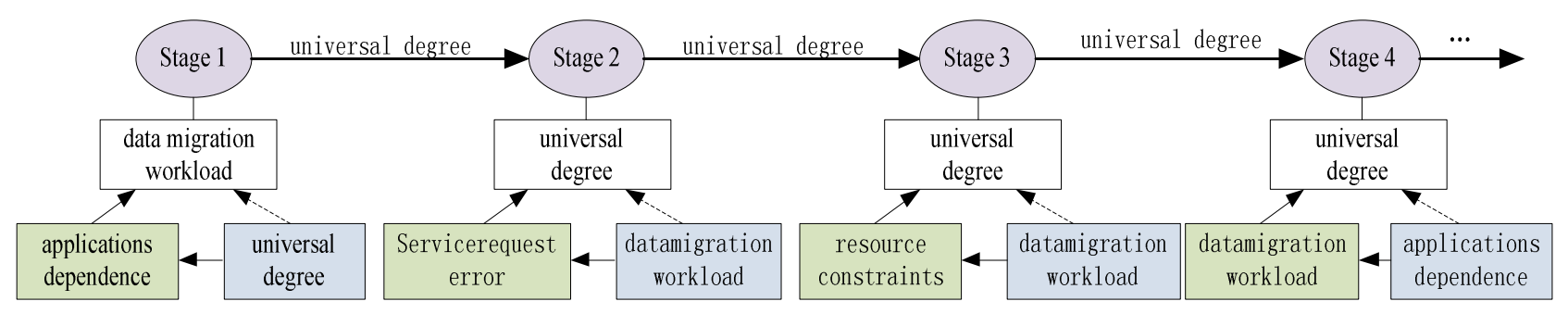

Figure 5. Cloud migration evolution of the business park.

As shown above, in each stage, a target element is aimed at; and the element which has the heaviest direct or indirect influence on the goal becomes the focus of auxiliary construction; Showing a biggest change of influence to the goal in current stage, the element with a strongest changing power acts as the bridge between two adjacent stages. So the planning scheme of next stage can work out referring to this bridge. Step by step, this scheme between 2 stages combines to serve as a guide to plan the enterprise strategy. Other factors, such as policy, economy and business background, all need to be fully considered in reality. Only the key development trend of enterprise is truly grasped can a strategic plan agreed with enterprise be worked out. 


\section{Conclusion and perspective}

This paper starts from the structure of ANP and analysis the influence process of element interaction to lead the enterprise evolution. The key elements vector sequence extracted from the process of initial state to the limit are used to improve the enterprise evolution strategy. Although the process of elements' influence on each other being discussed based on the matrix structure, its corresponding degree with enterprise development needs further proof. We will study further to explore the relationship between them.

\section{REFERENCES}

Andrew, R. B., Robert, N, \& Saaty, T.L., et al. (2002). Forecasting the resurgence of the US economy in 2001: an expert judgment approach Socio-Economic Planning Sciences. The Statistician, 36(2),77-91.

Davoudi, M.R., \& Sheykhvand, K. (2011). Enterprise Architecture Analysis using AHP and Fuzzy AHP. Proceedings of 2011 4th IEEE International Conference on Computer Science and Information Technology(ICCSIT 2011), VOL07,202-207

Ross, J.W. , Weill,P \& Robertson, D (2006). Enterprise architecture as strategy: Creating a foundation for business execution. Boston, Massachusetts: Harvard Business School Press.

Saaty, T.L. (1996). Decision making with dependence and feedback. Pittsburgh, PA: RWS Publications.

Ulutas, B.H. (2005). Determination of the appropriate energy policy for Turkey.Energy, 30(7),1146-1161

Wolfslehner, B., Vacik, H. \& Lexer, M.J. (2005). Application of the analytic network process in multicriteria analysis of sustainable forest management. Computers and Industrial Engineering, 207,157-170.

Xu, J.L., Liu, L., \& Shao, Z.Z. (2011). Research on the evolution of enterprise clusters based on theory of MAS. Proceedings of 2011 4th IEEE International Conference on Computer Science and Information Technology(ICCSIT 2011), VOL01,676-680

Ye, H.Y., \& Zhen, Z. (2012). Research on mechanism of enterprise evolution based on biological evolution. Science and Technology Management Research, 6, 218-221

Yang, X., \& Liu, M. (2011). Model of biding evaluation based on algorithm of adjusting weight of decision-makers. Engineering Education and Management, 53-61. 